\title{
Retrospective audit of the value of the pancreolauryl test in a district general hospital
}

\author{
I A Murray, S Clenton, B A McGeorge, A F Safe
}

Postgrad Med J 2003;79:471-473

See end of article for authors' affiliations

Correspondence and reprints requests to: Dr A F Safe, Northern General Hospital, Herries Road, Sheffield S5 7AU, UK; amirsafe@hotmail.com

Submitted

22 October 2002

Accepted

3 February 2003

\begin{abstract}
Objectives: To audit the specificity and value of the pancreolauryl test (PLT) for the diagnosis of pancreatic insufficiency.

Design: A retrospective case note review of 47 patients who had a PLT during three consecutive years. Setting: A 650 bedded district general hospital.

Main outcome measures: Patient demographics, symptoms, baseline blood, endoscopic and radiological investigations, and alcohol consumption were related to the results of the PLT to determine if the test could be better targeted. Response to therapy and final diagnoses were noted to determine the specificity of the test.

Results: Forty seven patients had a PLT during the three years studied. All successfully performed the test as outpatients. Seven inconclusive tests were repeated. Thirty eight patients $(81 \%)$ had diarrhoea, of whom $10(21 \%)$ described steatorrhoea; $23(49 \%)$ had an abnormal PLT. All with an abnormal test had diarrhoea, more frequently describing watery stools than steatorrhoea. Those treated with pancreatic enzyme supplements had an excellent response, although two of these had a final diagnosis of coeliac disease. Nineteen patients with an abnormal PLT were thought to be suffering from pancreatic insufficiency. All patients with a normal PLT were clinically thought not to have pancreatic insufficiency. Age, sex, and alcohol intake correlated poorly with final diagnosis.

Conclusions: The PLT can be used in a district general hospital setting as an outpatient test for pancreatic insufficiency and has a specificity of $83 \%-91 \%$. Its use should be restricted to thse presenting with diarrhoea, not necessarily steatorrhoea, and not restricted to those with a history of high alcohol consumption.
\end{abstract} he diagnosis of chronic pancreatitis depends on evidence of permanently abnormal pancreatic morphology, impaired function, or both. ${ }^{1}$ The best available methods for the study of pancreatic exocrine capacity and assessment of pancreatic insufficiency are tests requiring duodenal intubation with measurement of pancreatic enzyme and bicarbonate secretion. ${ }^{2}$ However, these tests are expensive, time consuming, and invasive. Faecal fat analysis is also time consuming, requires special laboratory equipment, and is distasteful to the patient, ward, and laboratory staff. The indirect (tubeless) pancreatic function tests, for example, para-amonibenzoic acid test and pancreolauryl test (PLT) have been increasingly used to assess for pancreatic insufficiency, particularly in nonspecialist units. They are easily performed by the patient, require standard laboratory equipment for analysis, are not expensive and have a high negative predictive value. ${ }^{34}$ The British Society of Gastroenterology guidelines for investigation of suspected malabsorption suggest direct tests should be restricted to specialist units and that PLT and paraamonibenzoic acid test are adequate screening tests.

Over the last few years, the PLT has been used in our unit to assess cases of chronic diarrhoea with suspected malabsorption. We carried out an audit of the use of the PLT to determine:

(1) The frequency of abnormal tests and whether subsequent clinical events matched these results.

(2) If any symptoms or simple blood tests predict an abnormal PLT.

(3) If PLT could be better targeted.

\section{METHODS}

\section{Patients}

Patients were identified from the hospital records system and pathology laboratory reports. Retrospective analysis of patients case notes was performed by two independent investigators.
The patients' demographic details, symptoms (diarrhoea, steatorrhoea, abdominal pain, and weight loss), alcohol intake, biochemical and haematological blood tests, radiologi$\mathrm{cal}$ and endoscopy investigations with relevant histology were recorded.

The audit included all PLTs performed over a three year period.

\section{Statistics}

Results are expressed as mean (SD). Regression analysis was performed to determine the significance of relations between clinical parameters and test results. A p value of $<0.05$ was considered significant.

\section{RESULTS}

Forty seven patients, who had a PLT over a three year period, were included in the audit. The age range was $17-76$ years ( 48 (13) years). Twenty six were male (55\%). Seven patients had a repeat test for equivocal results. Alcohol consumption was recorded in 40 patients (mean 33.1 units/week) with 11 men and five women were taking more than safe recommended limits (28 units/week and 21 units/week respectively).

Thirty eight patients $(81 \%)$ had diarrhoea of three months to 12 years' duration, of whom 10 described stools suggestive of steatorrhoea. The remainder had watery stools. Twenty two patients $(47 \%)$ had abdominal pain of three months to several years' duration. Nineteen patients had a weight loss of 1-5 stones.

Twenty three patients (49\%) had an abnormal PLT. Ten of 21 females (48\%) and 13 of 26 males (50\%) had abnormal results. Table 1 shows the number of abnormal and normal PLT results in relation to the patients' symptoms. Age was not correlated with PLT results (fig 1) and alcohol intake was poorly correlated (fig 2). 
Table 1 Relation between the pancreolauryl test (PLT) and patients' symptoms

\begin{tabular}{llllll}
\hline & $\begin{array}{l}\text { Diarrhoea } \\
(n=38)\end{array}$ & $\begin{array}{l}\text { Watery diarrhoea } \\
(n=28)\end{array}$ & $\begin{array}{l}\text { Steatorrhoea } \\
(n=10)\end{array}$ & $\begin{array}{l}\text { Weight loss } \\
(n=19)\end{array}$ & $\begin{array}{l}\text { Pain } \\
(n=22)\end{array}$ \\
\hline Abnormal PLT & 21 & 17 & 4 & 10 & 10 \\
Normal PLT & 17 & 11 & 6 & 9 & 12 \\
\hline
\end{tabular}

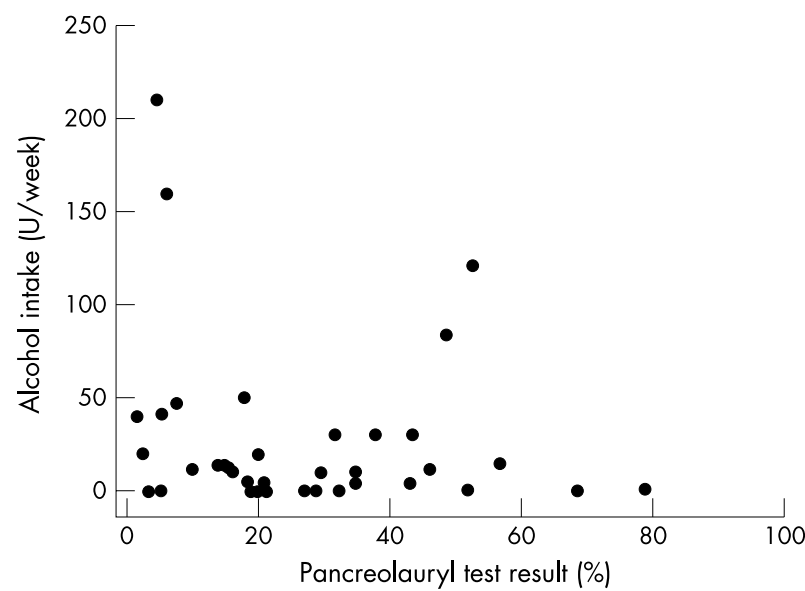

Figure 2 Relation between alcohol intake and pancreolauryl test results (abnormal ratio $<20 \%$ ).

Of the 28 patients with watery diarrhoea, $17(61 \%)$ had an abnormal PLT while four of 10 patients with steatorrhoea $(40 \%)$ had an abnormal test. In the absence of diarrhoea, weight loss and abdominal pain were not associated with an abnormal PLT.

Table 2 shows the biochemical results in relation to the PLT. Abnormal full blood count, calcium, and haematinics tended to predict an abnormal PLT but normal results did not indicate normal pancreatic function. Liver function tests were not predictive of the PLT result.

Duodenal biopsies were performed in 12 of the abnormal PLT patients. Two showed villous atrophy. Eleven patients with an abnormal PLT had an abdominal ultrasound, of whom seven $(64 \%)$ had structural pancreatic damage.

All the patients with normal PLT were thought clinically not to have pancreatic insufficiency. Nineteen of the 23 patients an with abnormal PLT were thought to be suffering from pancreatic malabsorption. Two with an abnormal PLT had a final diagnosis of coeliac disease.

Eighteen patients had an excellent response to pancreatic enzyme supplements (Creon), with marked improvement in

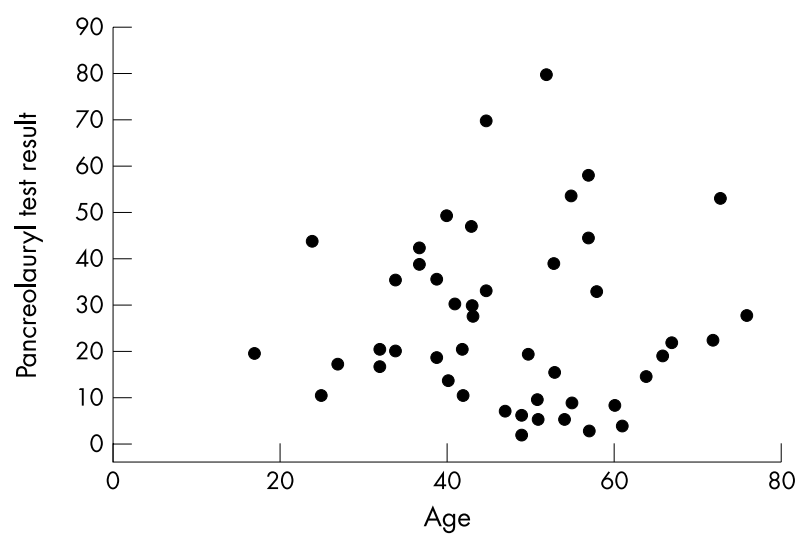

Figure 1 Relation between age and pancreolauryl test results (abnormal ratio $<20 \%$ ).

\begin{tabular}{|c|c|c|c|}
\hline \multicolumn{4}{|c|}{$\begin{array}{l}\text { Table } 2 \text { Pancreolauryl test (PLT) results and blood } \\
\text { tests results }\end{array}$} \\
\hline & \multirow[b]{2}{*}{$\begin{array}{l}\text { Abnormal } \\
\text { PLT/total }\end{array}$} & \multicolumn{2}{|c|}{ Abnormal PLT } \\
\hline & & $\begin{array}{l}\text { Positive } \\
\text { test }\end{array}$ & $\begin{array}{l}\text { Negative } \\
\text { test }\end{array}$ \\
\hline Full blood count & $10 / 47$ & $7 / 10$ & $14 / 37$ \\
\hline Liver function tests & $12 / 42$ & $7 / 13$ & $11 / 29$ \\
\hline Calcium & $1 / 32$ & $1 / 1$ & $15 / 31$ \\
\hline Haematinics & $3 / 31$ & $3 / 3$ & $12 / 28$ \\
\hline
\end{tabular}

the diarrhoea and weight gain. One patient improved on no therapy and another patient failed to attend for follow up.

\section{DISCUSSION}

Our audit demonstrates that the PLT can be a useful test to screen for pancreatic insufficiency in a district general hospital unit. Twenty three out of 47 patients $(49 \%)$ had abnormal results, of whom 19 were believed to be suffering from pancreatic malabsorption. On the other hand, all patients with a normal PLT were thought not have pancreatic insufficiency, indicating a high specificity.

Diarrhoea was the most common presentation in the group with an abnormal PLT. Interestingly, watery stools were more frequently associated with a positive test than steatorrhoea. Abnormal results were not seen with other presentatios (weight loss and abdominal pain) in the absence of diarrhoea. Males and females were equally likely to have an abnormal PLT and alcohol history did not predict the result.

Two patients with abnormal PLT results were found to have coeliac disease. Their abnormal result could be due to concomitant pancreatic insufficiency ${ }^{67}$ or to fat malabsorption secondary to villous atrophy. Forty percent of newly diagnosed coeliac patients have a positive PLT while only $18 \%$ of those on a gluten-free diet had a positive test, demonstrating that fat malabsorption may respond to diet. ${ }^{8}$ As the PLT was not repeated after starting a gluten-free diet, it is not known if these two tests were "false positives". Low serum calcium and haematinics with an abnormal PLT should prompt distal duodenal biopsies. The PLT has a high negative predictive value in the patients with coeliac disease and small bowel disease without pancreatic dysfunction. ${ }^{9}$

Abdominal ultrasound was not requested for all patients because it has poor sensitivity and is operator dependent in the investigation of pancreatic disease. ${ }^{10}$ Abdominal computed tomography, endoscopic retrograde cholangiopancreatography, or magnetic resonance cholangiopancreatography may be more appropriate, although many cases of pancreatic insufficiency are associated with normal imaging. ${ }^{.1}$

All patients who had an abnormal PLT received pancreatic enzyme supplements (Creon) and showed marked improvement on follow up. Although the majority did not have further imaging or direct (tube) tests, their response to treatment was considered sufficient to confirm the diagnosis of pancreatic insufficiency.

The PLT was easy for patients to carry out. Verbal instrutions were given to each patient by the laboratory and armacy staff, 
complemented by a typewritten instruction sheet. The test was suitable for use with outpatients as has previously been demonstrated ${ }^{12}$ and can be easily repeated.

The audit was carried out for a period of three years (199698 ) before faecal pancreatic elastase was widely used. We are now conducting another audit to compare both tests in investigating pancreatic insufficiency in the unit over the last two years.

The diagnosis of early chronic pancreatitis can be difficult and sometimes patients with pancreatic insufficiency may not present with classic symptoms. Although this audit confirms the high specificity of the PLT in a district general hospital setting, it does not determine the sensitivity. This has previously been reported as $30 \%-50 \%$ for mild pancreatic insufficiency with minimal anatomical changes on endoscopic retrograde cholangiopancreatography to $>95 \%$ for severe chronic pancreatitis. ${ }^{13}{ }^{14}$ It should be emphasised that the PLT would not exclude chronic pancreatitis in patients who do not have loss of exocrine pancreatic function.

The diagnosis of pancreatic insufficiency should be considered in all patients with unexplained chronic diarrhoea and weight loss. Pancreatic insufficiency may be seen in the absence of steatorrhoea and without a history of high alcohol intake. This audit shows that the PLT is a simple, inexpensive, and non-invasive screening test with specificity of $83 \%-91 \%$ for diagnosing pancreatic insufficiency in a district general hospital setting.

Authors' affiliations

I A Murray, S Clenton, A F Safe, Department of Gastroenterology, Barnsley District General Hospital, Barnsley, South Yorkshire

B A McGeorge, Department of Clinical Audit

\section{REFERENCES}

1 Malfertheiner $\mathbf{P}$, Büchler M. Correlation of imaging and function in chronic pancreatitis. Radiol Clin North Am 1989;27:51-63.

2 Boyd EJS, Wormsley KG. Laboratory tests in the diagnosis of chronic pancreatic diseases. 1. Secretagogues used in tests of pancreatic secretion. Int J Pancreatol 1987:2:137-48.

3 Boyd EJS, Cumming JGR, Cuschieri A, et al. Prospective comparison of the fluorescein-dilaurate test with secretin-cholecystokinin tests for pancreatic exocrine function. J Clin Pathol 1982;35:1240-3.

4 Lankisch PG, Schreiber A, Otto J. Pancreolauryl test. Evaluation of a tubeless pancreatic function test in comparison with other indirect and direct tests for exocrine pancreatic function. Dig Dis Sci 1983;28:490-3.

5 British Society of Gastroenterology. Tests for malabsorption. Loughborough: QSR, 1996.

6 Regan PT, Di Magno EP. Exocrine pancreatic insufficiency in coeliac disease: a cause for treatment failure. Gastroenterology 1980;78:484-7.

7 Ansaldi N, Oderda G. Exocrine pancreatic insufficiency in coeliac disease (letter). Gastroenterology 1981;80:883

8 Stevens FM, Kearns MC, McCarthy CF. Abnormal pncreolauryl tests in coeliac disease: lack of correlation with the degree of intestinal mucosal damage. J Clin Pathol 1997;50:1001-4.

9 Barry RE. Fluorescein dilaurate test (letter). Lancet 1985;ii:96.

10 Swobodnik W, Meyer W, Brecht-Kraus D, et al. Ultrasound, computed tomography and endoscopic retrograde cholangiopancreatography in the morphologic diagnosis of pancreatic disease. Klin Wochenschr 1983;61:291-6.

11 Lankisch PG, Seidensticker F, Otto J, et al. Secretin-pancreozymin test (SPT) and endoscopic retrograde cholangiopancreatography (ERCP): both are necessary for diagnosing or excluding chronic pancreatitis. Pancreas 1996;12:149-52.

12 Gould SR, Chinn GL, Nobbs BT, et al. Evaluation of a tubeless pancreatic function test in patients with steatorrhoea in a district general hospital. J R Soc Med 1988;819:270-3.

13 Domínguez-Muñoz JE, Hieronymus C, Sauerbruch T, et al. Fecal elastase test: evaluation of a new noninvasive pancreatic function test. Am J Gastroenterol 1995:90:1834-7.

14 Glasbrenner B, Schön A, Klatt S, et al. Clinical evaluation of the faecal elastase test in the diagnosis and staging of chronic pancreatitis. Eur $J$ Gatroenterol Hepatol 1996;8: $1117-20$ 